Sharif University of Technology
Scientia Iranica
SCIENTIA
I RAN I C A

\title{
A one-parameter controlled dissipative unconditionally stable explicit algorithm for time history analysis
}

\author{
S.-Y. Chang*, N.-C. Tran, T.-H. Wu and Y.-S. Yang \\ Department of Civil Engineering, National Taipei University of Technology, NTUT Box 2653, No. 1, Section 3, Jungshiau East \\ Road, Taipei 10608, Taiwan, Republic of China.
}

Received 9 December 2015; received in revised form 12 May 2016; accepted 25 July 2016

\author{
KEYWORDS \\ Unconditional \\ stability; \\ Numerical dissipation; \\ Explicit method; \\ Nonlinear dynamic \\ analysis; \\ Accuracy; \\ Structure-dependent \\ integration method.
}

\begin{abstract}
A new family of one-step integration methods is presented herein. A free parameter is used to control the numerical properties and it can be considered as an indicator of numerical dissipation for the high-frequency modes. This family of methods can have unconditional stability, explicit formulation, and desired numerical damping, which implies that the low-frequency modes can be accurately integrated while the spurious growth of high-frequency modes can be suppressed or even eliminated. In addition, a zero damping ratio can be achieved. Since the unconditional stability and explicit formulation are integrated for the proposed method family, it can drastically reduce the computational efforts when compared with the traditional integration methods.
\end{abstract}

(C) 2017 Sharif University of Technology. All rights reserved.

\section{Introduction}

Many dissipative integration methods have been proposed for structural dynamics, such as the Houbolt method [1], Newmark method [2], Wilson- $\theta$ method [3], HHT- $\alpha$ method [4], WBZ- $\alpha$ method [5], generalized- $\alpha$ method [6], Bathe method [7], Zhou and Tamma methods [8-9], Rezaiee-Pajand and Alamatian method [10], Gholampour and Ghassemieh method [11], and quartic $B$-spline method [12]. All these integration methods are implicit and, thus, they will involve an integration procedure for each time step in conducting time integration. It is well recognized that the nonlinear iterations for each time step will cost many computational efforts. On the other hand, if an integration method is explicit $[2,10,13,14]$, it has conditional stability; thus, a very small time-step size may be required to satisfy

\footnotetext{
*. Corresponding author. Tel.: +886-2-2771-2171;

Fax: +886-2-2781-4518

E-mail address: changsy@ntut.edu.tw (S.-Y. Chang)
}

the upper stability limit. Consequently, it will be very promising if these dissipative integration methods can be enhanced with an explicit formulation for implementation. The structure-dependent integration method was first developed by Chang in 2002 [15]. Some integration methods of this type were also successfully developed by Chang [16-22] subsequently. These structure-dependent integration methods can simultaneously integrate the major advantages of the implicit and explicit algorithms, i.e. the unconditional stability of implicit algorithms and no nonlinear iterations of explicit algorithms. However, they possess no numerical dissipation.

It is valuable to propose an integration method that possesses the desired numerical properties, which are unconditional stability, second-order accuracy, explicit formulation, and favorable numerical dissipation. Two family methods [20,23] have been developed for this purpose. However, they are two-step methods and, thus, a distinct starting procedure is generally needed for practical applications. Both family methods are expressed by the three parameters $\alpha, \beta$, and $\gamma$ and their numerical properties are dominated by these three 
parameters. In this work, a new family method is proposed. This family method is a one-step method and, thus, it is self-starting. In addition, its numerical properties are controlled by a free parameter, $p$, which can be considered as an indicator variable for numerical dissipation. The numerical properties of this family method are evaluated herein and some numerical examples are examined to confirm the analytical results.

\section{Proposed family method}

In structural dynamics or earthquake engineering, the equation of motion of the discrete model can be expressed by a set of second-order ordinary differential equations as:

$$
m \ddot{u}+c \dot{u}+k u=f,
$$

where $m, c, k$, and $f$ are the mass, viscous damping coefficient, stiffness, and external force, respectively; and $u, \dot{u}$, and $\ddot{u}$ are the displacement, velocity, and acceleration, respectively. This equation can be solved by many available step-by-step integration methods. A new family method is also proposed for solving this equation of motion. In order to develop the new family method, some basic assumptions are made for this development. Since the structure-dependent difference equation for displacement increment plays a key role to integrate the unconditional stability and explicit formulation, it is adopted. In addition, this difference equation is assumed to be a function of data of the previous step only since a one-step method is supposed to be developed. On the other hand, an asymptotic form of the equation of motion is assumed since it has been applied to develop some dissipative integration methods such as HHT- $\alpha$ method and WBZ$\alpha$ method. As a result, the proposed family method can be expressed as:

$$
\begin{gathered}
\frac{2}{p+1} m a_{i+1}+\frac{p-1}{p+1} m a_{i}+c v_{i+1}+k_{i+1} d_{i+1} \\
=f_{i+1} \\
d_{i+1}=d_{i}-B_{1} \Omega_{i}^{2} d_{i}+B_{2}(\Delta t) v_{i}+B_{3}(\Delta t)^{2} a_{i}, \\
v_{i+1}=v_{i}+\frac{3 p-1}{2(p+1)}(\Delta t) a_{i} \\
-\frac{p-3}{2(p+1)}(\Delta t) a_{i+1}
\end{gathered}
$$

where $d_{i}, v_{i}, a_{i}$, and $f_{i}$ are the nodal displacement, velocity, acceleration, and external force at the $i$ th time step, respectively; $\Delta t$ is step size and $k_{i}$ is the stiffness at the end of the $i$ th time step. In addition, $\Omega_{i}=$ $\omega_{i}(\Delta t)$; and $\omega_{i}=\sqrt{k_{i} / m}$ is the natural frequency of the system at the end of the $i$ th time step, $k_{i}$. Notice that $c v_{i+1}+k_{i+1} d_{i+1}$ in the first line of Eq. (2) can be rewritten as $N\left(v_{i+1}, d_{i+1}\right)$ for a general nonlinear system [24]. The coefficients $B_{1}$ to $B_{3}$ are:

$$
\begin{aligned}
& B_{1}=\frac{1}{4 D}\left(\frac{2}{p+1}\right)^{2} \\
& B_{2}=\frac{1}{D}\left\{\frac{2}{p+1}+\left[\frac{3-p}{p+1}-\frac{1}{2}\left(\frac{2}{p+1}\right)^{2}\right] \xi \Omega_{0}\right\} \\
& B_{3}=\frac{1}{D}\left\{\frac{p}{(p+1)^{2}}-\frac{1}{2}\left(\frac{p-1}{p+1}\right)^{2} \xi \Omega_{0}\right\}
\end{aligned}
$$

where:

$$
D=\frac{2}{p+1}+\frac{3-p}{p+1} \xi \Omega_{0}+\left(\frac{1}{p+1}\right)^{2} \Omega_{0}^{2}
$$

where $\xi$ is a viscous damping ratio; $\Omega_{0}=\omega_{0}(\Delta t)$; and $\omega_{0}=\sqrt{k_{0} / m}$ is the natural frequency of the system determined from the initial stiffness of $k_{0}$. Notice that $\Omega_{i}=\Omega_{0}$ and $\omega_{i}=\omega_{0}$ are found for $i=1,2,3, \ldots$ for a linear elastic system. The development details of this method are similar to those of the previously published algorithms [17,18] and, thus, they will not be elaborated on herein. In this derivation, the proof of convergence must be conducted first. Hence, the requirements of the order of accuracy and unconditional stability of the proposed family method are used to determine the coefficients $B_{1}$ to $B_{3}$ appropriately. This work is very complicated. The order of accuracy is determined from the local truncation error of the proposed family method. On the other hand, the procedure given by Lambert [25] and then invoking the Routh-Hurwitz criterion can be applied to determine the unconditional stability. Notice that $p$ is the free parameter to govern the numerical properties. It will be shown later that $p$ can be considered as the spectral radius of the proposed family method in the limit $\Omega_{0} \rightarrow \infty$ for a linear elastic system.

For computational efficiency, it is very important to rewrite $\xi \Omega_{0}$ and $\Omega_{0}^{2}$ in terms of the initial structural properties and step size for a structure-dependent integration method. Thus, based on the theory of structural dynamics, the relations $\Omega_{0}^{2}=(\Delta t)^{2}\left(k_{0} / m\right)$ and $c_{0}=2 \xi \omega_{0} m$ can be obtained as it is assumed that viscous damping ratio, $c_{0}$, is determined from the initial structural properties. After substituting these relations into Eqs. (3) and (4), they become:

$$
B_{1}=\frac{1}{D} \frac{1}{(p+1)^{2}} m
$$




$$
\begin{aligned}
& B_{2}=\frac{1}{D}\left\{\frac{2}{p+1} m-\frac{\left(p^{2}-2 p-1\right)}{2(p+1)^{2}}(\Delta t) c_{0}\right\}, \\
& B_{3}=\frac{1}{D}\left\{\frac{2}{(p+1)^{2}} m-\frac{1}{4}\left(\frac{p-1}{p+1}\right)^{2}(\Delta t) c_{0}\right\}, \\
& D=\frac{2}{p+1} m+\frac{3-p}{2(p+1)}(\Delta t) c_{0}+\left(\frac{1}{p+1}\right)^{2}(\Delta t)^{2} k_{0} .
\end{aligned}
$$

Notice that the coefficients $B_{1}, B_{2}$, and $B_{3}$ depend only on the initial properties of the structure and step size. Hence, they will remain invariant and, thus, there is no need to re-compute these coefficients during a whole step-by-step integration procedure.

\section{Recursive matrix form}

Since the numerical properties of PFM can be derived from the characteristic equation of its amplification matrix, it is needed to rewrite Eq. (2) in a recursive matrix form. Thus, the use of PFM to obtain the free vibration in a system with single degree of freedom can be expressed in the following form:

$$
\mathbf{X}_{i+1}=\mathbf{A} \mathbf{X}_{i}=\mathbf{A}^{(i+1)} \mathbf{X}_{0}
$$

where $\mathbf{X}_{i+1}=\left[d_{i+1},(\Delta t) v_{i+1},(\Delta t)^{2} a_{i+1}\right]^{T}$ is defined and $\mathbf{A}$ is an amplification matrix. Notice that the vector $\mathbf{X}_{0}$ is in correspondence with the given initial conditions $d_{0}$ and $v_{0}$. The initial acceleration can be determined by $a_{0}=\left(f_{0}-c v_{0}-k d_{0}\right) / m$. The explicit expression of the amplification matrix $A$ for a linear elastic system is found as shown in Box I, where $B$ is further defined as:

$$
B=\frac{2}{p+1}+\frac{3-p}{p+1} \xi \Omega_{0} .
$$

Thus, the characteristic equation of the amplification matrix, $\mathbf{A}$, can be obtained from $|\mathbf{A}-\lambda \mathbf{I}|=0$ and is found to be:

$$
\lambda^{3}-A_{1} \lambda^{2}+A_{2} \lambda-A_{3}=0
$$

where $\lambda$ is an eigenvalue of the amplification matrix $\mathbf{A}$ and the coefficients $A_{1}, A_{2}$, and $A_{3}$ are found to be:

$$
\begin{aligned}
A_{1}= & -2 p+\frac{3 p+5}{(p+1) D}+\frac{2(2-p)}{D} \xi \Omega_{0}, \\
A_{2}= & -p+2+\frac{\left(-p^{2}+2 p-5\right)}{(p+1) D} \xi \Omega_{0} \\
& +\frac{(p-1)(p+2)}{(p+1)^{2} D} \Omega_{0}^{2} \\
A_{3}= & -\frac{(p-1)}{(p+1) D} .
\end{aligned}
$$

Notice that the characteristic equation can be applied to evaluate the numerical properties of PFM.

\section{Convergence}

The convergence of a computational method is implied by the consistency and the stability based on the Lax equivalence theorem [24]. In general, the consistency is defined by a qualitative measure, such as the order of accuracy, which can be directly determined from the local truncation error. In general, an algorithm is said to be convergent if it is both consistent and stable.

\subsection{Consistency and local truncation error}

A local truncation error is defined as the error committed in each time step by replacing the differential equation with its corresponding difference equation [26-28]. The approximating difference equation for PFM can be obtained from Eq. (6) after eliminating velocities and accelerations and is found to be:

$$
d_{i+1}-A_{1} d_{i}+A_{2} d_{i-1}-A_{1} d_{i-2}=0 \text {. }
$$

Consequently, after replacing Eq. (1) by Eq. (11), the local truncation error for PFM is:

$$
\begin{aligned}
E= & \frac{1}{(\Delta t)^{2}}\left[u(t+\Delta t)-A_{1} u(t)+A_{2} u(t-\Delta t)\right. \\
& \left.-A_{3} u(t-2 \Delta t)\right] .
\end{aligned}
$$

In addition, if $u(t)$ is assumed to be continuously differentiable up to any required order, the terms of

$$
\mathbf{A}=\frac{1}{D}\left[\begin{array}{ccc}
B\left(1-B_{1} \Omega_{0}^{2}\right) & B^{*} B_{2} & B^{*} B_{3} \\
\frac{p-3}{2(p+1)}\left(1-B_{1} \Omega_{0}^{2}\right) \Omega_{0}^{2} & \frac{2}{p+1}-\frac{3-p}{2(p+1)} B_{2} \Omega_{0}^{2} & \frac{1}{2}-\frac{3-p}{2(p+1)} B_{3} \Omega_{0}^{2} \\
-\left(1-B_{1} \Omega_{0}^{2}\right) \Omega_{0}^{2} & -2 \xi \Omega_{0}-B_{2} \Omega_{0}^{2} & -\frac{p-1}{p+1}-\frac{3 p-1}{p+1} \xi \Omega_{0}-B_{3} \Omega_{0}^{2}
\end{array}\right]
$$


$u(t+\Delta t), u(t-\Delta t)$, and $u(t-2 \Delta t)$ can be expanded into finite Taylor series at $t$. As a result, after substituting $A_{1}, A_{2}$, and $A_{3}$ into the result of Eq. (11), the local truncation error for PFM is found to be:

$$
\begin{aligned}
E_{i+1}= & -\frac{\Omega_{0}^{2} \omega_{0}^{2}}{12(p+1)^{2} D}\left\{\left[4(p+1)(5 p-7) \xi^{2}\right.\right. \\
& \left.-\left(p^{2}+2 p-7\right)\right] u_{i}+4\left[2(p+1)(5 p-7) \xi^{2}\right. \\
& \left.\left.-3\left(p^{2}-3\right)\right] u_{i}\right\}+O\left[(\Delta t)^{3}\right]
\end{aligned}
$$

for a linear elastic system. This equation reveals that PFM has the minimum order of accuracy of 2 if Rayleigh damping is adopted and, thus, its consistency is verified for any values of $p$ and any viscous damping ratio $\xi$.

\subsection{Stability}

The stability analysis of PFM is very difficult by using an algebraic method. This is because it has three nonzero eigenvalues due to $A_{3} \neq 0$. Alternatively, the stability conditions will first be considered for the two especial cases of $\Omega_{0} \rightarrow 0$ and $\Omega_{0} \rightarrow \infty$ and, then, a general value of $\Omega_{0}$ is studied. After finding the coefficients $A_{1}, A_{2}$, and $A_{3}$ for the case of $\Omega_{0} \rightarrow 0$, Eq. (9) becomes:

$$
(\lambda-1)^{2}\left(\lambda+\frac{p-1}{2}\right)=0 .
$$

This equation reveals that PFM has two principal roots of $\lambda_{1,2}=1$ in addition to a spurious root of $\lambda_{3}=-(p-$ $1) / 2$. Apparently, these eigenvalues are independent of $\xi$. To satisfy the stability condition, the value of $\lambda_{3}$ must be in the interval of $-1 \leq \lambda_{3} \leq 1$. As a result, $-1 \leq p \leq 3$ is obtained. Similarly, in the limit of $\Omega_{0} \rightarrow \infty$, Eq. (9) is reduced to:

$$
\lambda(\lambda+p)^{2}=0
$$

where two principal roots are $\lambda_{1,2}=-p$ and the spurious root is $\lambda_{3}=0$. This implies that $-1<p \leq 1$ must be met for any viscous damping ( $p$ cannot be -1 because $p=-1$ leads some factor to be infinity).

After obtaining the range of $-1<p \leq 1$ for PFM to have unconditional stability in the limiting cases of $\Omega_{0} \rightarrow 0$ and $\Omega_{0} \rightarrow \infty$, it is needed to further confirm that if the same range is applicable to a general value of $\Omega_{0}$. This can be evaluated by using the Routh-Hurwitz criterion, which gives necessary and sufficient condition for the roots of Eq. (9) to lie within or on the circle of $|\lambda|=1$ if the following inequalities are satisfied:

$$
\begin{aligned}
& 1-A_{1}+A_{2}-A_{3} \geq 0, \quad 3-A_{1}-A_{2}+3 A_{3} \geq 0 \\
& 3+A_{1}-A_{2}-3 A_{3} \geq 0, \quad 1+A_{1}+A_{2}+A_{3} \geq 0 \\
& 1-A_{2}+A_{3}\left(A_{1}-A_{3}\right) \geq 0 .
\end{aligned}
$$

After substituting Eq. (10) into Eq. (16), it is found that all the five inequalities will be met if $-1<p \leq 1$ holds; this proves the stability of PFM. This stable property in conjunction with the previous proof of consistency implies the convergence of PFM.

\section{Numerical properties}

It is generally recognized that the spectral radius, relative period error, numerical damping, and overshooting are the numerical properties of PFM, which will be further investigated herein. The techniques for evaluating these numerical properties can be found in the references $[26,28,29]$ and, thus, will not be elaborated on here.

\subsection{Spectral radius}

The variations of spectral radii with $\Delta t / T_{0}$, where $T_{0}=$ $2 \pi / \omega_{0}$, are shown in Figure 1 for $p=1.0,0.5,0,-0.5$, and -0.99 . The spectral radius is the maximum absolute eigenvalue of the amplification matrix. For a small value of $\Delta t / T_{0}$, the spectral radius is almost equal to 1.0 for each curve; while, for a larger value of $\Delta t / T_{0}$, it decreases gradually and tends to a certain value. Notice that it is always equal to 1.0 for $p=1$. This means that PFM can have zero damping for $p=1$. At first glance, it seems that the value of $p$ can be chosen to be either in the range of $-1<p \leq 0$ or $0 \leq p \leq 1$ since both ranges can provide appropriate numerical damping. However, it is worth noting that the curve for $p=-0.5$ shows an abrupt change of slope at the point $(0.24,0.62)$ as shown in Figure 1 . This point might be a bifurcation point, where complex conjugate roots bifurcate into real roots.

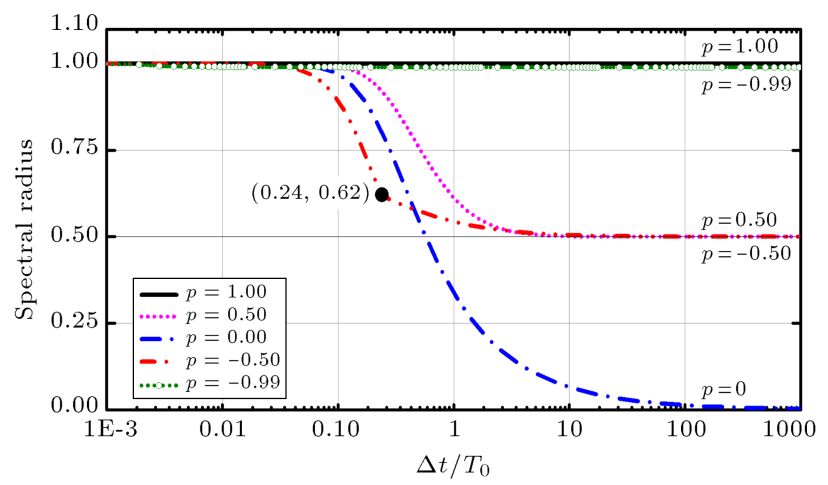

Figure 1. Variations of spectral radius with $\Delta t / T_{0}$ for PFM. 


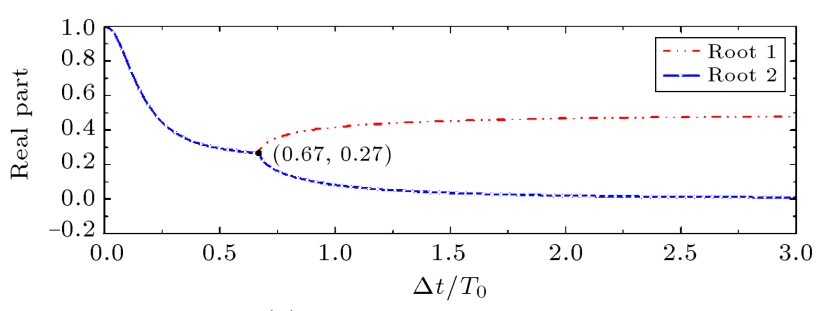

(a) Real part of principal root

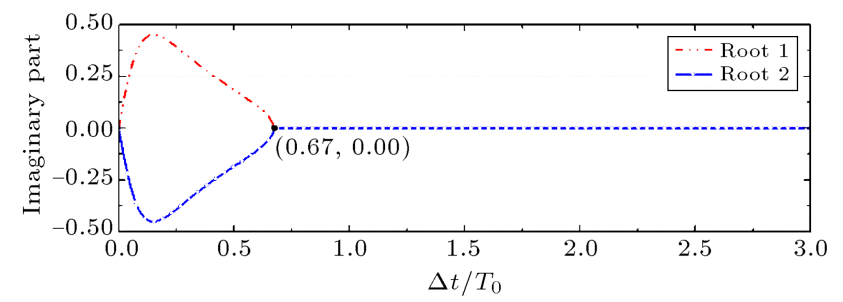

(b) Imaginary part of principal root

Figure 2. Principal roots of PFM with $p=-0.5$.

In order to clarify the difference between the ranges of $-1<p \leq 0$ and $0 \leq p \leq 1$ with respect to period distortion, the eigenvalues of PFM for the case of $p=-0.5$ are calculated and plotted in Figure 2 . It is evident that the imaginary part of each principal root will become zero after the value of $\Delta t / T_{0}$ grows greater than around 0.67 , as marked by a solid circle in the figure. This confirms that the complex conjugate roots bifurcate into real roots at this bifurcation point. In general, the two real roots imply that the obtained response is in an exponential decay form and there is no bounded oscillatory response, which is generally preferred for an integration method. Further calculations reveal that a bifurcation point is generally found in the range of $-1<p<0$, which is of no interest for practical applications. Accordingly, the following study will focus on the range of $0 \leq p \leq 1$ for PFM.

\subsection{Relative period error}

Figure 3 illustrates the relative period error of $\left(\bar{T}_{0}-\right.$ $\left.T_{0}\right) / T_{0}$ against $\Delta t / T_{0}$ for $p=1.0,0.75,0.75$, and 0.0. In addition, the results for the HHT- $\alpha$ method and WBZ- $\alpha$ method are also plotted in this figure for comparison. In general, the relative period error

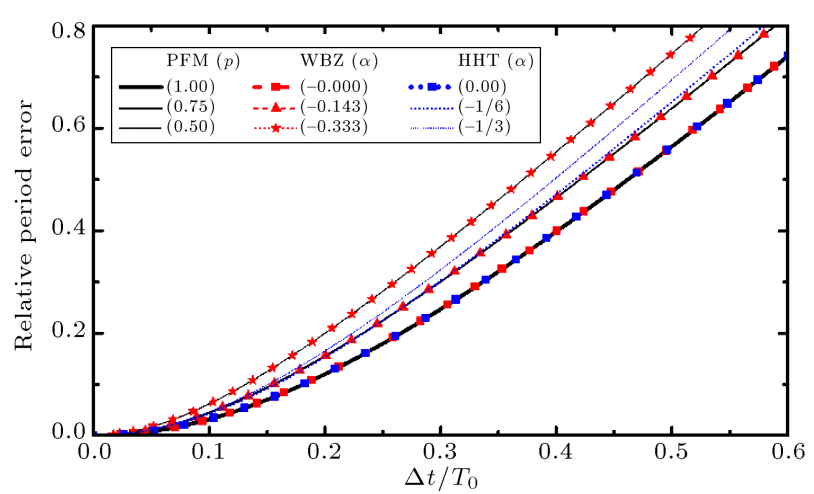

Figure 3. Variations of relative period error with $\Delta t / T_{0}$ for PFM. increases with increase in $\Delta t / T_{0}$ for each curve. It is interesting to note that the curve of PFM with $p=1$ almost coincides with that of HHT and WBZ as $\alpha=0$. This phenomenon is likely to be found for PFM with $p=0.75$, HHT with $\alpha=-\frac{1}{6}$, and WBZ with $\alpha=-0.143$. Although the curve of PFM with $p=0$ seems to overlap with that of WBZ with $\alpha=-0.333$, both methods show more period distortion than that of HHT with $\alpha=-\frac{1}{3}$. However, the difference in period distortion among the three family methods is not very significant for a small value of $\Delta t / T_{0}$, say $\Delta t / T_{0} \leq 0.05$.

It is found that a large value of $p$ will lead to a small relative period error for a given value of $\Delta t / T_{0}$. It has been considered as a good rule of thumb for choosing $\Delta t / T_{0} \leq 0.05$ to yield a reliable response [27]. This criterion indicates that the range of $0.5 \leq p \leq 1$ might be of great interest for practical applications, since PFM with a $p$ value in the range of $0 \leq p \leq 0.5$ seems to result in too much period distortion.

\subsection{Numerical damping}

The numerical damping ratio can be applied to evaluate the numerical dissipation of a time integration method Figure 4. shows the variation of numerical damping ratio versus $\Delta t / T_{0}$ for $\mathrm{PFM}$ with $p=1,0.75$, and 0.0. For each curve, the numerical damping ratio is very small for a small value of $\Delta t / T_{0}$ and, then, it increases gradually; finally, it becomes constant. In this figure, it is also found that the numerical damping ratio is controlled by the $p$ value only, where $p=0$ gives the highest numerical damping ratio and $p=1$ leads to zero damping. In general, a large value of $p$ value will result in a small numerical damping ratio for a given value of $\Delta t / T_{0}$. This figure also implies that PFM with a $p$ value in the range of $0 \leq p \leq 0.5$ may lead to very large numerical dissipation for low frequency modes since PFM with $p=0$ has a numerical damping ratio of $2 \%$ for $\Delta t / T_{0}=0.05$.

For comparison, the results for HHT and WBZ are also plotted in Figure 4. In general, zero damping

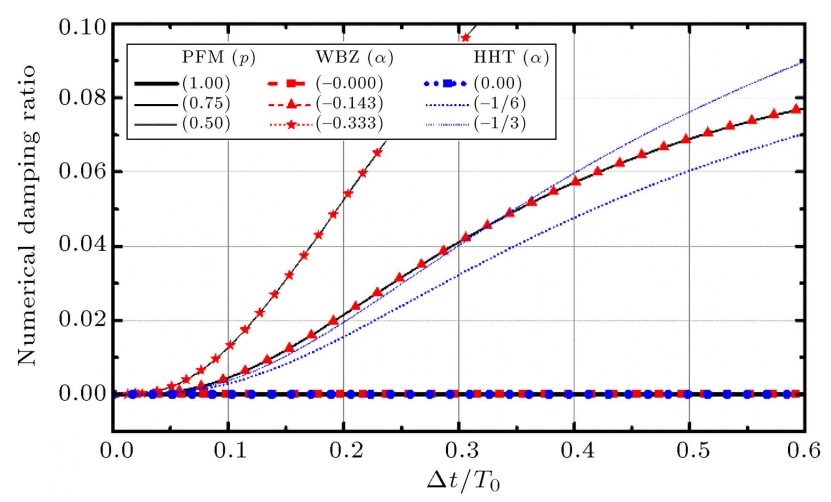

Figure 4. Variations of numerical damping ratio with $\Delta t / T_{0}$ for different values of $p$. 
is achieved for PFM with $p=1$, HHT with $\alpha=0$, and WBZ with $\alpha=0$. It is also found that the curves for PFM with $p=0.75$ and 0.50 almost overlap with those for WBZ with $\alpha=-0.143$ and $\alpha=-0.333$, respectively. Notice that PFM with $p=0.5$ and WBZ with $\alpha=-0.333$ will result in the maximum numerical damping ratios, which are generally larger than those of HHT with $\alpha=-\frac{1}{3}$.

In Figure 1, it is found that in the limit of $\Omega_{0} \rightarrow \infty$ (or $\Delta t / T_{0} \rightarrow \infty$ ), the spectral radius is convergent on the $p$ value. In fact, the spectral radii for $p=1.0,0.5$, and 0.0 are found to be $1.0,0.5$, and 0.0 , respectively, in the limit of $\Omega_{0} \rightarrow \infty$. On the other hand, Figure 4 reveals that a large $p$ value leads to a small numerical damping ratio. Consequently, the free parameter, $p$, might be considered as an indicator variable of numerical dissipation for high-frequency modes. As a result, a value of $p$ close to 1 implies a small numerical damping for high-frequency modes while a value of $p$ close to 0 indicates a large numerical damping for high-frequency modes. In summary, a small $p$ value will lead to a large numerical damping ratio while it accompanies a large relative period error.

\subsection{Overshooting}

In order to evaluate the tendency of an integration method to overshoot the exact solutions in the early response [30,31], the free vibration response of a linear elastic single-degree-of-freedom model problem is often considered. In general, it can be expressed as:

$$
\ddot{u}(t)+\omega^{2} u(t)=0
$$

with initial conditions $u(0)=d_{0}$ and $\dot{u}(0)=v_{0}$. Since $\mathrm{PFM}$ is a converged method, there is no overshoot as $\Omega_{0} \rightarrow 0$. In general, $\Omega_{0} \rightarrow \infty$ can provide an indication of the overshooting behavior of the highfrequency mode in a system where the values of $\Delta t / T_{0}$ are large for the high-frequency mode. As a result, the following equations can be obtained for the limiting condition of $\Omega_{0} \rightarrow \infty$ :

$$
\begin{aligned}
& d_{i+1} \approx-p d_{i} \\
& v_{i+1} \approx-\frac{1}{4}(p-1)^{2} \Omega_{0}^{2}\left(\frac{d_{i}}{\Delta t}\right)+\left[\frac{1}{2}(p-1)^{2}-1\right] v_{i}
\end{aligned}
$$

The first line of this equation reveals that there is no overshooting in displacement for any member of PFM while it has a tendency to overshoot quadratic $\Omega_{0}$ in the velocity equation due to the initial displacement term as indicated by the second line of this equation (U0-V1). Interestingly, the overshooting velocity disappears and becomes U0-V0 for $p=1$.

In order to confirm the analytical prediction of the overshooting behavior of PFM, the free vibration
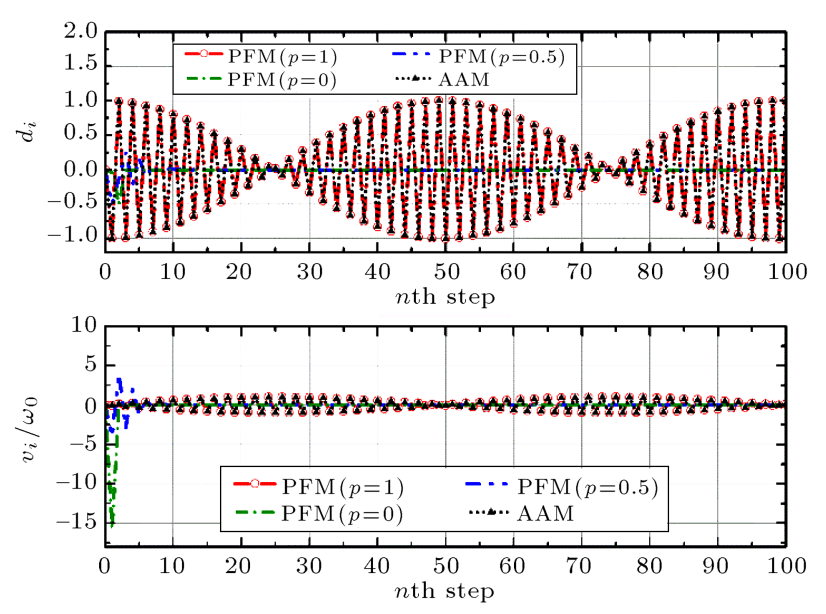

Figure 5. Comparison of overshooting responses.

response of a single-degree-of-freedom system is calculated by using a relatively large time step. In fact, it is computed by using PFM with $p=1,0.5$, and 0 and AAM with a time step corresponding to $\Delta t / T_{0}=10$ for the initial conditions of $d_{0}=1$ and $v_{0}=0$. Numerical results are shown in Figure 5, where the velocity term is normalized by the natural frequency of system in order to have the same unit as that of displacement. The horizontal axis measures time in a number of time steps. It is manifested in the top plot of Figure 5 that all the three curves for PFM exhibit no overshoot in displacement. In addition, the curve for PFM with $p=$ 1 coincides with that of AAM. A significant overshoot in velocity is found in the bottom plot of this figure for $p=0.5$ and 0 although it is almost annihilated in the first few time steps. Again, the two curves for PFM with $p=1$ and AAM overlap. This implies that PFM with $p=1$ has the same overshooting behaviors as those of AAM for a linear elastic system. As a result, these numerical results are in good agreement with the analytical results.

\section{Implementation details}

For the applications of PFM to solve a multiple-degreeof-freedom system, the implementation of PFM is presented next. The general formulation for PFM can be written as:

$$
\begin{gathered}
\left(\frac{2}{p+1}\right) \mathbf{M} \mathbf{a}_{i+1}+\left(\frac{p-1}{p+1}\right) \mathbf{M} \mathbf{a}_{i}+\mathbf{C}_{0} \mathbf{v}_{i+1} \\
+\mathbf{K}_{i+1} \mathbf{d}_{i+1}=\mathbf{f}_{i+1}, \\
\mathbf{d}_{i+1}=\left[\mathbf{I}-\mathbf{B}_{1}\left(\mathbf{M}^{-1} \mathbf{K}_{i}\right)(\Delta t)^{2}\right] \mathbf{d}_{i}+(\Delta t) \mathbf{B}_{2} \mathbf{v}_{i}+(\Delta t)^{2} \mathbf{B}_{3} \mathbf{a}_{i}, \\
\mathbf{v}_{i+1}=\mathbf{v}_{i}+(\Delta t)\left[\frac{3 p-1}{2(p+1)} \mathbf{a}_{i}+\frac{3-p}{2(p+1)} \mathbf{a}_{i+1}\right],
\end{gathered}
$$

where $\mathbf{M}$ is a mass matrix, $\mathbf{C}_{0}$ is a constant damping 
matrix and is assumed to be determined from the initial structural properties, and $\mathbf{K}_{i}$ is the stiffness matrix at the end of the $i$ th time step; $\mathbf{d}_{i}, \mathbf{v}_{i}, \mathbf{a}_{i}$, and $\mathbf{f}_{i}$ are the nodal vectors of the displacement, velocity, acceleration, and external force, respectively; and $\mathbf{I}$ is an identity matrix with the size of $n \times n$, where $n$ is the total number of degrees of freedom of the system. The coefficient matrices of $\mathbf{B}_{1}$ to $\mathbf{B}_{3}$ and $\mathbf{D}$ are found to be:

$$
\begin{aligned}
& \mathbf{B}_{1}=\frac{1}{(p+1)^{2}} \mathbf{D}^{-1} \mathbf{M} \\
& \mathbf{B}_{2}=\mathbf{D}^{-1}\left[\frac{2}{p+1} \mathbf{M}-\frac{\left(p^{2}-2 p-1\right)}{2(p+1)^{2}}(\Delta t) \mathbf{C}_{0}\right] \\
& \mathbf{B}_{3}=\mathbf{D}^{-1}\left[\frac{2}{(p+1)^{2}} \mathbf{M}-\frac{(p-1)^{2}}{4(p+1)^{2}}(\Delta t) \mathbf{C}_{0}\right] \\
& \mathbf{D}=\frac{2}{p+1} \mathbf{M}+\frac{3-p}{2(p+1)}(\Delta t) \mathbf{C}_{0}+\frac{1}{(p+1)^{2}}(\Delta t)^{2} \mathbf{K}_{0}
\end{aligned}
$$

where $\mathbf{K}_{0}$ is the initial stiffness matrix of the system. It is generally different from the stiffness matrix, $\mathbf{K}_{i}$, in Eq. (19) for a nonlinear system. It is important to note that the coefficient matrices $\mathbf{B}_{1}$ to $\mathbf{B}_{3}$ must be determined from the initial structural properties of $\mathbf{M}$, $\mathbf{C}_{0}$, and $\mathbf{K}_{0}$ as well as the step size before performing the time integration.

At the start of the $(i+1)$-th time step, the displacement vector can be computed by using the second line of Eq. (19). It is numerically equivalent to the outcome of following equation:

$$
\begin{aligned}
\mathbf{D}\left(\mathbf{d}_{i+1}\right. & \left.-\mathbf{d}_{i}\right)=-\frac{1}{(p+1)^{2}}(\Delta t)^{2} \mathbf{r}_{i} \\
& +\left[\frac{2}{p+1} \mathbf{M}-\frac{\left(p^{2}-2 p-1\right)}{2(p+1)^{2}}(\Delta t) \mathbf{C}_{0}\right](\Delta t) \mathbf{v}_{i} \\
& +\left[\frac{p}{(p+1)^{2}} \mathbf{M}-\frac{(p-1)^{2}}{4(p+1)^{2}}(\Delta t) \mathbf{C}_{0}\right](\Delta t)^{2} \mathbf{a}_{i}
\end{aligned}
$$

After obtaining the current displacement vector, the assumed force-displacement relations can be applied to determine the corresponding restoring force vector. In general, $\mathbf{r}_{i+1}=\mathbf{K d}_{i+1}$ is used to represent the restoring force vector. Subsequently, the velocity vector, $\mathbf{v}_{i+1}$, can be calculated by substitution of the third line of Eq. (19) into the first line of this equation. As a result, the resultant equation is numerically equivalent to the outcome of the following equation:

$$
\left[\left(\frac{2}{p+1}\right) \mathbf{M}+\left(\frac{3-p}{2(p+1)}\right)(\Delta t) \mathbf{C}_{0}\right] \mathbf{v}_{i+1}
$$

$$
\begin{aligned}
& =\left(\frac{3-p}{2(p+1)}\right)(\Delta t)\left(\mathbf{f}_{i+1}-\mathbf{r}_{i+1}\right) \\
& +\left(\frac{2}{p+1}\right) \mathbf{M} \mathbf{v}_{i} \\
& +\left(\frac{5 p-3}{2(p+1)}\right)(\Delta t) \mathbf{M a}_{i}
\end{aligned}
$$

Finally, the acceleration vector, $\mathbf{a}_{i+1}$, can be directly obtained from the equations of motion and is numerically equivalent to:

$$
\begin{aligned}
\left(\frac{2}{p+1}\right) \mathbf{M} \mathbf{a}_{i+1}= & \mathbf{f}_{i+1}-\left(\frac{p-1}{p+1}\right) \mathbf{M} \mathbf{a}_{i} \\
& -\mathbf{C}_{0} \mathbf{v}_{i+1}-\mathbf{r}_{i+1}
\end{aligned}
$$

A direct elimination method is often applied to solve Eqs. (21) to (23). However, there is no need to apply a direct elimination method to solve Eq. (23) if $\mathbf{M}$ is a diagonal matrix. Similarly, Eq. (22) will involve no direct elimination methods if $\mathbf{M}$ is a diagonal matrix in addition to a zero damping matrix.

It is generally recognized that a direct elimination method is made up of a triangulation and a substitution for each time step and a triangulation will consume the most time in each time step. Since the coefficient matrices on the left-hand side of Eqs. (21) to (23) remain invariant during time integration, the triangulation of these coefficient matrices needs to be performed only once. On the other hand, this implementation involves no nonlinear iterations for each time step. Hence, it is anticipated to be computationally very efficient when compared to a traditional implicit integration method. Notice that the proposed family method may still have an explicit formulation if the external force vector is a function of displacement. This is because the external force vector can be determined after obtaining the displacement vector by using Eq. (21). However, it seems that an explicit formulation cannot be achieved in the case the internal force vector is a nonlinear function of velocity and/or displacement, since Eq. (22) cannot be used to determine the velocity vector due to the presence of the unknown restoring force vector.

\section{Numerical examples}

Analytical investigations reveal that PFM can have favorable numerical properties, such as unconditional stability, second-order accuracy, and desired numerical dissipation. Consequently, it is of great interest to examine its actual performance in the step-by-step solution of a dynamic problem. Hence, some numerical examples are particularly selected and solved for this purpose. In the following calculations, PFM with $p=1$ and 0.5 will be applied to perform the step-by-step 
integration. For brevity, PFM with $p=1$ is referred to as PFM1 and PFM with $p=0.5$ is referred to as PFM2. Notice that PFM1 has no numerical dissipation while PFM2 has favorable numerical dissipation. For comparison, both the Newmark Explicit Method (NEM) and AAM are also applied to solve the same problems.

\subsection{Example 1: Seismic responses to 6-story building}

The initial structural properties of a 6-story shearbeam type building are simulated by a 6-degree-offreedom system with its masses and initial stiffness as follows:

$$
\begin{aligned}
& m_{1}=m_{2}=10^{3} \mathrm{~kg}, \quad m_{3}=m_{4}=10^{5} \mathrm{~kg}, \\
& m_{5}=m_{6}=10^{8} \mathrm{~kg}, \quad k_{0-i}=10^{10} \mathrm{~N} / \mathrm{m} \\
& i=1 \sim 6 .
\end{aligned}
$$

The stiffness of each story is nonlinear behavior, which is assumed to be a function of the story drift. The stiffness for each story can be written in the form of:

$$
k_{j-i}=k_{0-i}\left[1+q_{i}\left(\left|u_{i}-u_{i-1}\right|\right)^{1 / 5}\right], \quad i=1 \sim 5
$$

where $k_{j-i}$ is the instantaneous stiffness for the $i$ th story at the end of the $j$ th time step and $k_{0-i}$ is the initial stiffness for the $i$ th story at the start of motion; $\left|u_{i}-u_{i-1}\right|$ is the story drift for the $i$ th story and $q_{i}$ is a given constant coefficient corresponding to the story drift. It is very straightforward to simulate a nonlinear system by simply specifying appropriate $q_{i}$ values. In fact, a nonlinear elastic system is simulated by choosing $q_{1}=-1.0$ and $q_{2}=q_{3}=\ldots=q_{6}=-0.5$. As a result, the mass matrix, $\mathbf{M}$, and stiffness matrix, $\mathbf{K}$, of the system can be expressed by Eqs. (26) and (27) as shown in Box II.

All the initial natural frequencies and the 1st and 6 th modal shapes of the building are calculated based on the initial properties and shown in the following:

$$
\begin{aligned}
& \omega_{1}=6.1758, \\
& \omega_{2}=16.176, \\
& \omega_{3}=194.21, \\
& \omega_{4}=510.22, \\
& \omega_{5}=1973.3, \\
& \omega_{6}=5119.4 \\
& \left.\phi_{1}=\left[\begin{array}{l}
0.617 \\
0.999 \\
1.000 \\
1.000 \\
1.000 \\
1.000
\end{array}\right], \quad(\mathrm{rad} / \mathrm{sec})\right)
\end{aligned}
$$

To confirm that PFM can be used to reliably calculate the response to a very complex earthquake load and that PFM has favorable numerical dissipation, the seismic response to a ground acceleration record with a different initial condition is also calculated for the building by AAM, PFM1, and PFM2. The building is excited by the ground acceleration record of $\mathrm{CHY} 028$ at its base, where the peak ground acceleration is scaled to $0.5 \mathrm{~g}$ for this study. This record was provided from the 1999 Chi-Chi earthquake in central Taiwan. Meanwhile, in order to illustrate the effectiveness of numerical damping, a high-frequency modal error is intentionally introduced into the calculated system through a given initial displacement, which consists of the 6 th mode only.

The three integration methods of AAM, PFM1, and PFM2 are used to compute the seismic responses with a time step of $\Delta t=0.01 \mathrm{sec}$. The numerical results for the top story responses are plotted in Figure 6. The result obtained from AAM subject to CHY028 without the high-frequency modal error is considered as a reference solution for comparison. On

$$
\begin{aligned}
& \mathbf{M}=\operatorname{diag}\left(m_{i}\right), \quad i=1,6 \\
& \mathbf{K}=\left[\begin{array}{cccccc}
k_{j-1}+k-j-2 & -k_{j-2} & & & \\
-k_{j-2} & k_{j-2}+k_{j-3} & -k_{j-3} & & \\
& -k_{j-3} & k_{j-3}+k_{j-4} & -k_{j-4} & \\
& & -k_{j-4} & k_{j-4}+k_{j-5} & -k_{j-5} & \\
& & & -k_{j-5} & k_{j-5}+k_{j-6} & -k_{j-6} \\
& & & -k_{j-6} & k_{j-6}
\end{array}\right]
\end{aligned}
$$




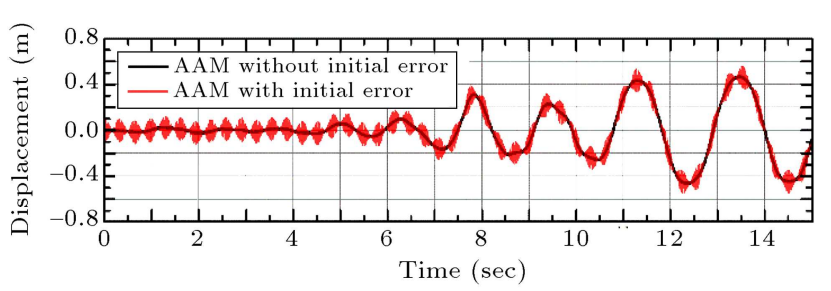

(a) AAM

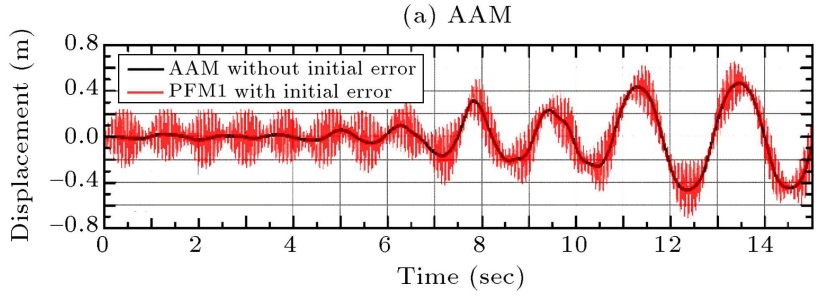

(b) PFM1

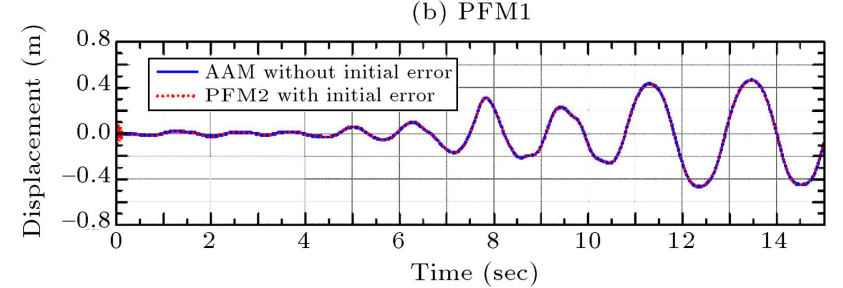

(c) PFM2

Figure 6. Displacement responses at the top of building.

the other hand, all the three integration methods are employed to compute the seismic responses to $\mathrm{CHY} 028$ with the high-frequency modal error. Figure 6(a) and (b) show that the results obtained from AAM and PFM1 are contaminated or even destroyed by the highfrequency modal error, while the result obtained from PFM2 in Figure 6(c) almost overlaps with the reference solution. It can be said that the time step of $\Delta t=$ $0.01 \mathrm{sec}$ is small enough to obtain accurate solutions for AAM and PFM2. Moreover, PFM2 has the favorable numerical dissipation and, thus, it can filter out the high-frequency responses within about $0.1 \mathrm{sec}$ for nonlinear systems, while both AAM and PFM1 do not have any numerical dissipation. Interestingly, the results, as shown in Figure 6(a) and (b), indicate that the response achieved from AAM is less contaminated than that from PFM1. This example also indicates that PFM can have unconditional stability since the value of $\Omega_{0}=\omega(\Delta t)$ for the 6 th mode is as large as 51.19. For comparison, it should be mentioned that the condition of stability for Newmark explicit method is $\Omega_{0} \leq 2$ for an undamped system [26].

\subsection{Example 2: Reinforced concrete frame subjected to a sinusoidal load}

A model of reinforced concrete frame with all of its dimensions, sections, and material properties is created and shown in Figure 7. The model consists of four nodes, of which the bottom two nodes are fixed, two column elements, and a beam element. The sections of
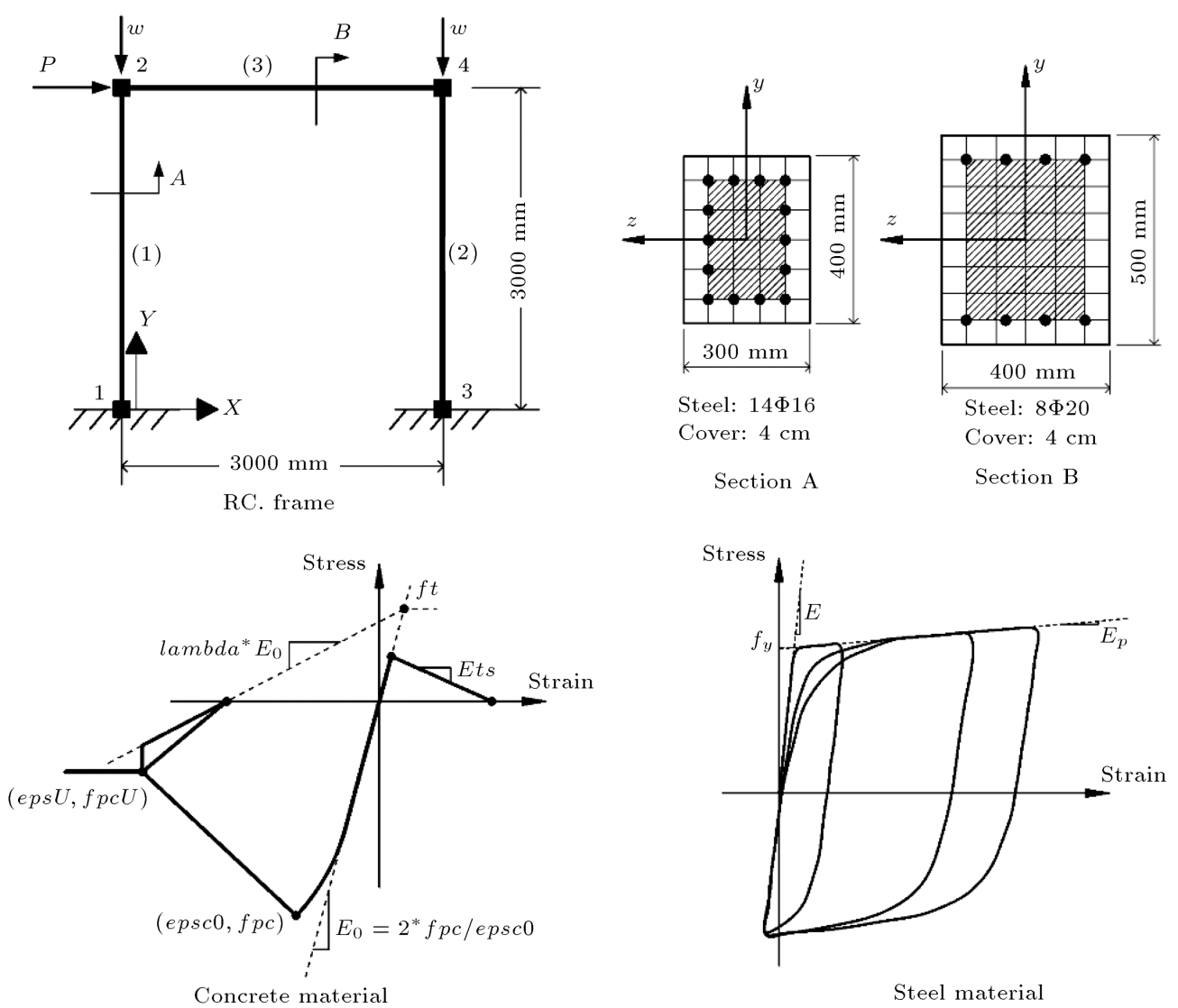

Figure 7. The reinforced concrete frame. 
beam and column are broken down into fibers where uniaxial materials are defined independently, which are shown in the figure. Concrete is defined as the uniaxial concrete material object with tensile strength and linear tension softening [32] while steel is defined as a material with isotropic strain hardening [33]. The $4 \mathrm{~cm}$ thick cover layer is considered as an unconfined concrete with lower strength than the confined concrete strength inside the bar area of elements. The properties of materials are described as follows:

Confined concrete:

$$
\begin{aligned}
& f_{c}=-286 \mathrm{~kg} / \mathrm{cm}^{2}, \quad \varepsilon_{c_{0}}=0.0019, \\
& f_{p c U}=-57.2 \mathrm{~kg} / \mathrm{cm}^{2}, \quad \varepsilon_{U}=-0.0095, \\
& \lambda=0.1, \quad f_{t}=40.4 \mathrm{~kg} / \mathrm{cm}^{2}, \\
& E_{t s f}=15400 \mathrm{~kg} / \mathrm{cm}^{2} .
\end{aligned}
$$

Unconfined concrete:

$$
\begin{aligned}
& f_{c}=-220 \mathrm{~kg} / \mathrm{cm}^{2}, \quad \varepsilon_{c_{0}}=-0.003, \\
& f_{p c U}=-44 \mathrm{~kg} / \mathrm{cm}^{2}, \quad \varepsilon_{U}=-0.01, \\
& \lambda=0.1, \quad f_{t}=30.8 \mathrm{~kg} / \mathrm{cm}^{2}, \\
& E_{t s f}=15400 \mathrm{~kg} / \mathrm{cm}^{2} .
\end{aligned}
$$

Steel:

$$
\begin{aligned}
& f_{y}=3600 \mathrm{~kg} / \mathrm{cm}^{2}, \\
& E=2 \times 10^{6} \mathrm{~kg} / \mathrm{cm}^{2}, \\
& E_{p}=0.05^{*} E .
\end{aligned}
$$

A reinforced concrete frame is loaded by a sinusoidal function, $P$, at its top in the $x$-direction under a constant weight, $w$, along the vertical direction. The constant weight is $200 \mathrm{kN}$ and the applied load pattern in $z$-direction is $P=200 \sin (\pi t) \mathrm{kN}$. The initial natural frequency of the first mode is found to be $2.886 \mathrm{rad} / \mathrm{sec}$ only based on the linear elastic stiffness matrix, whereas this is up to $9.1 \times 10^{5} \mathrm{rad} / \mathrm{sec}$ for the last mode. A time step of $\Delta t \leq 2.2 \times 10^{-6} \mathrm{sec}$ must be used to carry out the time integration for using NEM so that the upper stability limit can be met. Hence, AAM is used to replace NEM for calculating the reference solution with a time step of $\Delta t=0.01 \mathrm{sec}$. PFM1 with the same time step of $\Delta t=0.01 \mathrm{sec}$ is also applied to compute the responses.

The displacement responses at Node 2 of the frame along the $x$-direction are plotted in the top plot of Figure 8(a). The hysteretic loops of the outermost steel bar layer and concrete at the fixed end of column are recorded and shown in the bottom of Figure 8(b) and (c). In general, both AAM and PFM1 integration methods can provide reliable solutions to the very

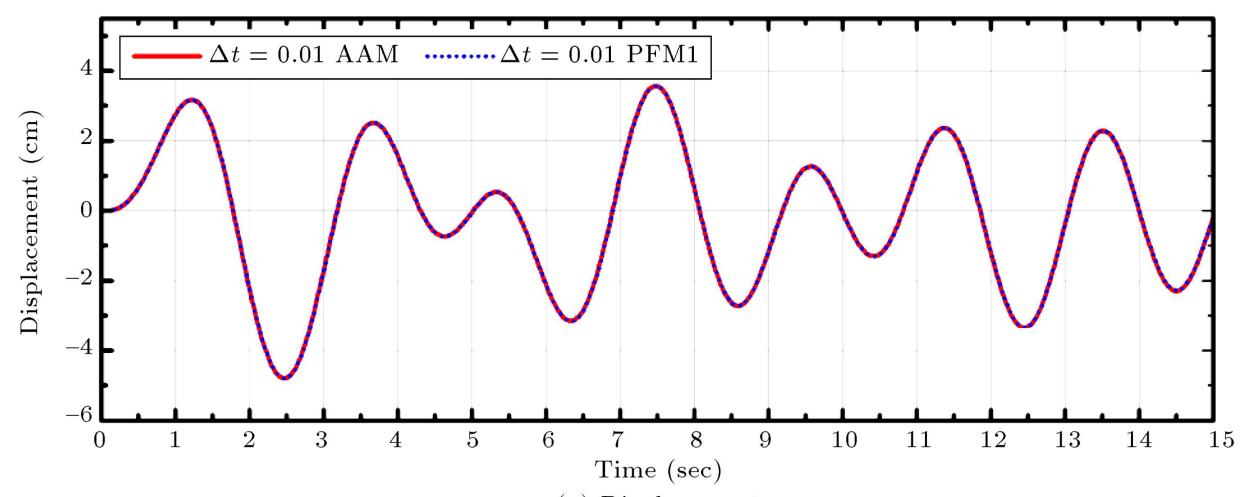

(a) Displacement

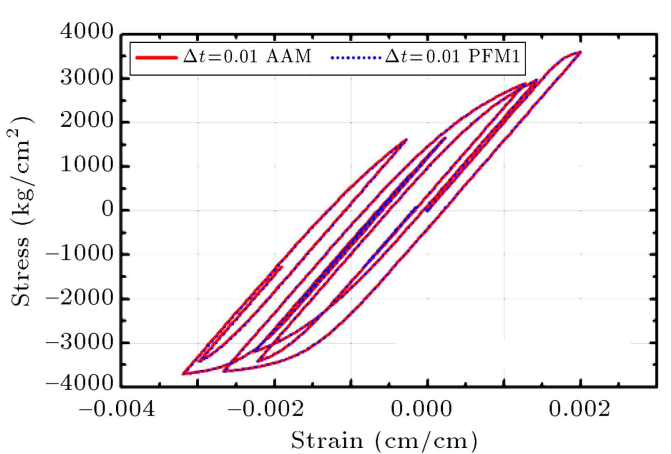

(b) Steel strain-stress curve

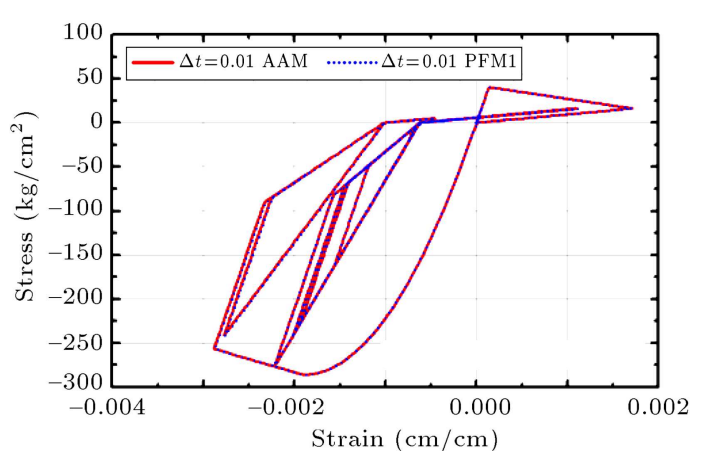

(c) Concrete strain-stress curve

Figure 8. Responses to sinusoidal load and corresponding hysteretic loops of materials. 
complex nonlinear problem as shown in Figure 7 . The bottom plot reveals that the material experiences a nonlinear behavior during the vibration for both concrete and steel. It is clearly indicated by this example that PFM can have unconditional stability since the highest frequency mode is as large as $9.1 \times 10^{5}$ $\mathrm{rad} / \mathrm{sec}$. In addition, the capability of using $\mathrm{PFM}$ to solve a highly nonlinear system is verified since it can result in a reliable solution without involving any iteration procedure in each time step.

\subsection{Example 3: Computational efficiency}

In order to study the computational efficiency of PFM, an $n$-degree-of-freedom spring mass system, as shown in Figure 9(a), is considered, where $m_{i}=1 \mathrm{~kg}$ and $k_{i}=$ $10^{8}\left(1-10 \sqrt{\left|u_{i}-u_{i-1}\right|}\right)(\mathrm{N} / \mathrm{m})$ for $i=1,2,3, \ldots, n$ are taken. Notice that $u_{i}$ is the displacement corresponding to the $i$ th spring mass (or the $i$ th degree of freedom). The responses to the 500-DOF $(n=500)$, 1000-DOF $(n=1000)$, and 2000-DOF $(n=2000)$ systems are computed by using NEM, AAM, and PFM2. These spring-mass systems are excited by the combinations of sine loads as shown in Figure 9(a). It is found that the lowest natural frequency of the 500-DOF system is $31.38 \mathrm{rad} / \mathrm{sec}$ before it deforms, while it is $15.70 \mathrm{rad} / \mathrm{sec}$ and $7.85 \mathrm{rad} / \mathrm{sec}$ for $1000-\mathrm{DOF}$ and $2000-$ DOF systems, respectively. On the other hand, the three systems have the same highest natural frequency equal to $20000.0 \mathrm{rad} / \mathrm{sec}$. A time step of $\Delta t=0.0001$ sec is chosen to follow the stability conditions for NEM, while the time step of $\Delta t=0.005 \mathrm{sec}$ is selected for AAM and PFM2 based on accuracy consideration. The running codes are written in Fortran ${ }^{\circledR}$, and the execution platform is a personal computer with Intel ${ }^{\circledR}$ Core $^{\mathrm{TM}}$ i5 CPU M460 @ $2.53 \mathrm{GHz}$ with installed memory of (RAM) 4.00 GB.

The displacement response time histories of the three systems are calculated and shown in Figure 9(b). The numerical solution obtained from NEM with a very small time step is considered as the reference solution. In general, PFM2 can have reliable solutions with comparable accuracy to AAM. The CPU time consumed by each of the aforementioned methods in the analysis is recorded and summarized in Table 1 . It is interesting to note that the CPU time consumed by PFM2 is about $10 \%$ to $20 \%$ of that consumed by NEM, and only about $2 \%$ to $3 \%$ of that consumed by AAM. This is because

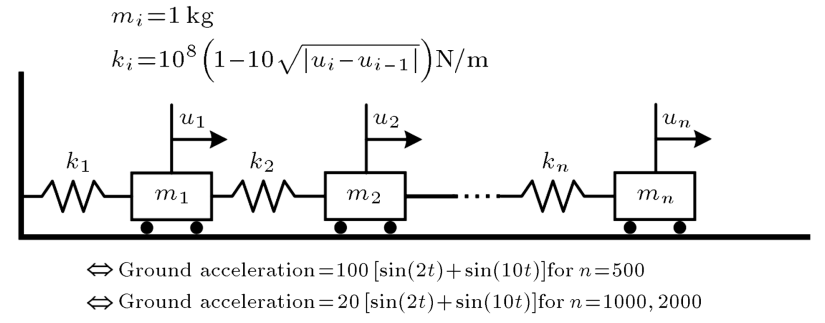

(a) An $n$-degree-of-freedom system
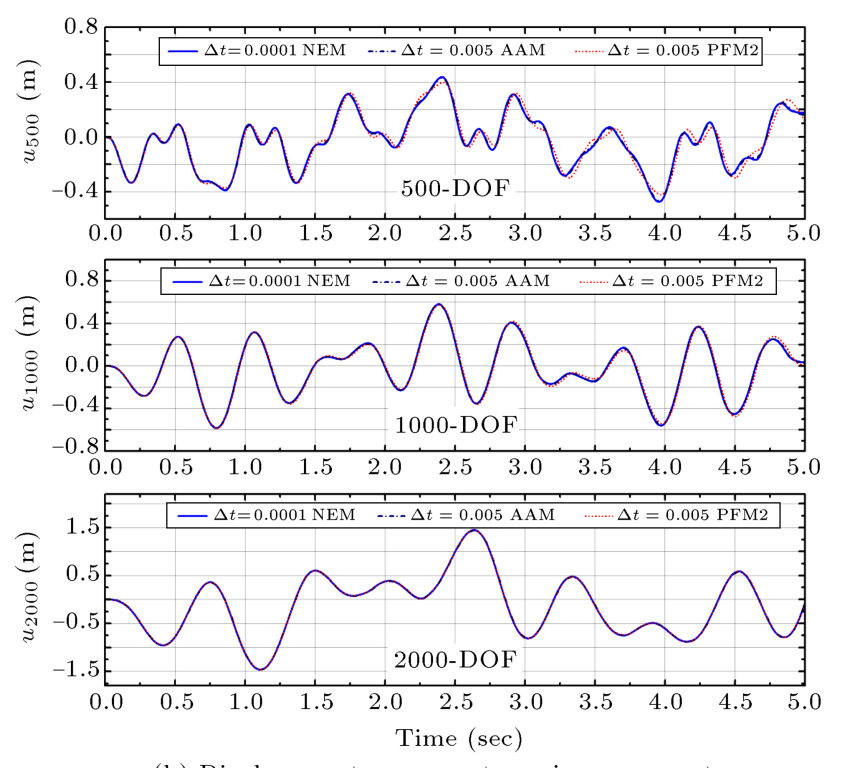

(b) Displacement response to spring-mass system

Figure 9. The spring-mass systems and their responses.

PFM2 can have the unconditional stability and, thus, there is no constraint on selecting an appropriate step size based on stability consideration. Notice that a very small step size is adopted for NEM since it is only conditionally stable. On the other hand, PFM2 can have an explicit formulation and, thus, it involves no nonlinear iteration for each time step and can save many computational efforts. Apparently, an iteration procedure, which is very time consuming for a matrix of a large order, is needed in each time step for AAM since AAM is an implicit method although it possesses the unconditional stability.

\section{Conclusions}

A new family of explicit time integration methods is proposed herein. The numerical properties of the

Table 1. Comparison of CPU times.

\begin{tabular}{|c|c|c|c|c|c|}
\hline N-DOF & $\begin{array}{c}\mathrm{CPU}^{\text {(NEM) }} \\
(\mathbf{1})\end{array}$ & $\begin{array}{c}\mathrm{CPU}^{(\mathrm{AAM})} \\
(2)\end{array}$ & $\begin{array}{c}\mathrm{CPU}^{(\mathrm{PFM2})} \\
(3)\end{array}$ & $(3) /(1)$ & $(3) /(2)$ \\
\hline 500 & 478.48 & 1532.67 & 45.32 & 0.095 & 0.030 \\
\hline 1000 & 1588.48 & 13567.28 & 302.66 & 0.191 & 0.022 \\
\hline 2000 & 2058.10 & 21429.36 & 442.50 & 0.215 & 0.021 \\
\hline
\end{tabular}


proposed family method are controlled by a free parameter, $p$. This family method is a one-step method and, thus, it is a self-starting integration method. In addition, it has unconditional stability, secondorder accuracy, explicit formulation, and favorable numerical dissipation. The value of $p$ ranges from 0 to 1 . As the $p$ value is close to 1 , it leads to low numerical damping for high-frequency modes and a zero numerical damping ratio can be achieved for $p=1$. On the other hand, as the $p$ value is close to 0 , it results in high numerical damping for high-frequency modes and, thus, the spurious participation of highfrequency modes can be suppressed or eliminated while the low-frequency modes can be accurately integrated. This free parameter, $p$, can be considered as an indicator variable of numerical damping for high-frequency modes. In addition, the range of $0.5 \leq p \leq 1$ is highly recommended for practical application since the range of $0<p<0.5$ may result in too much period distortion and numerical dissipation for the low-frequency modes. The computational efficiency of this family method is evident from the numerical experiments when compared to the conventional integration methods. This is mainly because it can integrate the unconditional stability with explicit formulation.

\section{Acknowledgment}

The authors acknowledge the financial support of this study by the National Science Council, Taiwan, R.O.C., under Grant No. NSC-100-2221-E-027-062.

\section{References}

1. Houbolt, J. "A recurrence matrix solution for the dynamic response of elastic aircraft", Journal of Aeronautical Science, 17, pp. 540-550 (1950).

2. Newmark, N. "A method of computation for structural dynamics", Journal of Engineering Mechanics Division, ASCE, 85, pp. 67-94 (1959).

3. Wilson, E., Farhoomand, I. and Bathe, K.J. "Nonlinear dynamic analysis of complex structures", Earthquake Engineering and Structural Dynamics, 1, pp. 241-252 (1973).

4. Hilber, H., Hughes, T.J.R. and Taylor, R. "Improved numerical dissipation for time integration algorithms in structural dynamics", Earthquake Engineering and Structural Dynamics, 5, pp. 283-292 (1977).

5. Wood, W., Bossak, M. and Zienkiewicz, O.C. "An alpha modification of Newmark's method", International Journal for Numerical Methods in Engineering, 15, pp. 1562-1566 (1980).

6. Chung, J. and Hulbert, G. "A time integration algorithm for structural dynamics with improved numerical dissipation: The generalized- $\alpha$ method", Journal of Applied Mechanics, 60(6), pp. 371-375 (1993).
7. Bathe, K.J. and Baig, M.M. "On a composite implicit time integration procedure for nonlinear dynamics", Computers \& Structures, 83(31-32), pp. 2513-2524 (2005).

8. Zhou, X. and Tamma, K.K. "Design, analysis, and synthesis of generalized single step single solve and optimal algorithms for structural dynamics", International Journal for Numerical Method in Engineering, 59, pp. 597-668 (2004).

9. Zhou, X. and Tamma, K.K. "Algorithms by design with illustrations to solid and structural mechanics/dynamics", International Journal for Numerical Methods in Engineering, 66, pp. 1738-1790 (2006).

10. Rezaiee-Pajand, M. and Alamatian, J. "Numerical time integration for dynamic analysis using a new higher order predictor-corrector method", Engineering Computations, 25(6), pp. 541-568 (2008).

11. Gholampour, A.A. and Ghassemieh, M. "Nonlinear structural dynamics analysis using weighted residual integration", Mechanics of Advanced Materials and Structures, 20(3), pp. 199-216 (2013).

12. Shojaee, S., Rostami, S. and Abbasi, A. "An unconditionally stable implicit time integration algorithm: Modified quartic B-spline method", Computers \& Structures, 153, pp. 98-111 (2015).

13. Rostami, S., Shojaee, S. and Saffari, H. "An explicit time integration method for structural dynamics using cubic B-spline polynomial functions", Scientia Iranica, 20(1), pp. 23-33 (2013).

14. Rezaiee-Pajand, M. and Karimi-Rad, M. "A new explicit time integration scheme for nonlinear dynamic analysis", International Journal of Structural Stability and Dynamics, 16(9), 1550054 (26 pages) (2015).

15. Chang, S.Y. "Explicit pseudodynamic algorithm with unconditional stability", Journal of Engineering Mechanics, ASCE, 128(9), pp. 935-947 (2002).

16. Chang, S.Y. "Improved explicit method for structural dynamics", Journal of Engineering Mechanics, ASCE, 133(7), pp. 748-760 (2007).

17. Chang, S.Y. "An explicit method with improved stability property", International Journal for Numerical Method in Engineering, 77(8), pp. 1100-1120 (2009).

18. Chang, S.Y. "A new family of explicit method for linear structural dynamics", Computers \& Structures, 88(11-12), pp. 755-772 (2010).

19. Chang, S.Y. "Family of structure-dependent explicit methods for structural dynamics", Journal of Engineering Mechanics, 140(6), 06014005 (7 pages) (2014a).

20. Chang, S.Y. "A family of non-iterative integration methods with desired numerical dissipation", International Journal of Numerical Methods in Engineering, 
100, pp. 62-86 (2014b).

21. Chang, S.Y. "A general technique to improve stability property for a structure-dependent integration method", International Journal for Numerical Methods in Engineering, 101(9), pp. 653-669 (2014c).

22. Chang, S.Y. "Comparisons of structure-dependent explicit methods for time integration", International Journal of Structural Stability and Dynamics, 15(3), 1450055 (20 pages) (2015a).

23. Chang, S.Y. "Dissipative, non-iterative integration algorithms with unconditional stability for mildly nonlinear structural dynamics", Nonlinear Dynamics, 79(2), pp. 1625-1649 (2015b).

24. Lax, P. and Richmyer, R. "Survey of the stability of linear difference equations", Communications on Pure and Applied Mathematics, 9, pp. 267-293 (1956).

25. Lambert, J.D., Computational Method in Ordinary Differential Equations, London: John Wiley (1973).

26. Belytschko, T. and Hughes, T.J.R. Computational Methods for Transient Analysis, North-Holland: Elsevier Science Publishers B.V. (1983).

27. Bathe, K.J., Finite Element Procedure in Engineering Analysis, Englewood Cliffs, NJ, USA.: Prentice-Hall, Inc. (1986).

28. Hughes, T.J.R., The Finite Element Method, Toronto, Canada: General Publishing Company Ltd. (1987).

29. Zienkiewicz, O.C., The Finite Element Method, London: McGraw-Hill (1977).

30. Goudreau, G. and Taylor, R. "Evaluation of numerical methods in elastodynamics", Journal of Computer Methods in Applied Mechanics and Engineering, 2, pp. 69-73 (1973).

31. Hilber, H. and Hughes, T.J.R. "Collocation, dissipation, and 'overshoot' for time integration schemes in structural dynamics", Earthquake Engineering and Structural Dynamics, 6, pp. 99-118 (1978).

32. Yassin, M.H.M. "Nonlinear analysis of prestressed concrete structures under monotonic and cycling loads", $\mathrm{PhD}$ Dissertation, University of California, Berkeley, California (1994).

33. Filippou, F.C., Popov, E.P. and Bertero, V.V. Effects of Bond Deterioration on Hysteretic Behavior of Reinforced Concrete Joints, Earthquake Engineering Research Center, University of California, Berkeley, California (1983).

\section{Biographies}

Shuenn-Yih Chang received a PhD degree from the University of Illinois, Urbana-Champaign, in 1995. He currently serves as a distinguished professor at the
National Taipei University of Technology. His research focuses on the structural dynamics and earthquake engineering. His work addresses dynamic testing of large-scale earthquake resistant structures, including the development of novel pseudo-dynamic techniques. $\mathrm{He}$ is also greatly interested in the design of accurate and efficient computational methods for dynamic problems of contemporary engineering. He has published more than one hundred refereed journal articles since 1988.

Ngoc-Cuong Tran received his BS and MS degrees from the Department of Civil Engineering at Hanoi Architectural University, Vietnam. He was awarded the Taiwan Government Scholarship for his $\mathrm{PhD}$ course. He received the $\mathrm{PhD}$ degree from the Department of Civil Engineering at the National Taipei University of Technology in 2016. His research interests are in the areas of structural analysis, structural dynamics, and nonlinear time-history analysis.

Tsui-Huang Wu received his BS degree from the Department of Civil Engineering at National Central University in 1979, his MS degree from the Civil Engineering Department at National Taiwan University in 1983, and his PhD from the Banking and Finance Department at Tamkang University in 2013. Currently, he is studying a PhD course in the Department of Civil Engineering at National Taipei University of Technology. His research interests are in the areas of designing and investigation of buildings damaged by fire, designing and safety assessment for civil and structural engineering in high-rise buildings, soil and structure interaction under earthquake loading, and structural analysis.

Yuan-Sen Yang is an Associate Professor at the National Taipei University of Technology. He also serves as a Committee Chairman of Information Committee of the Chinese Society of Structural Engineering in Taiwan. Before serving in university, he was a Research Associate in the National Center for Research on Earthquake Engineering. He received BS from National Chung-Hsing University in 1995 and $\mathrm{PhD}$ from National Taiwan University in 2000. His research interests center on image analysis of structural experiments, high-performance computing of nonlinear finite element analysis, and numerical modeling of nonlinear structural dynamics. His teaching includes computer programming, numerical analysis, engineering statistics, engineering materials, structural experiments, parallel computing, and scientific visualization. 